\title{
Aumento do índice de massa corporal após os 20 anos de idade e associação com indicadores de risco ou de proteção para doenças crônicas não transmissiveis
}

\author{
Body mass index increase after the age of 20 and associations with \\ risk or protection factors for chronic non-communicable diseases
}

Mara Sérgia Pacheco Honório Coelho', Maria Alice Altenburg de Assis', Erly Catarina Moura ${ }^{2,3}$

${ }^{1}$ Programa de Pós-Graduação em Nutrição, Centro de Ciências da Saúde, Universidade Federal de Santa Catarina (UFSC), Florianópolis, SC, Brasil ${ }^{2}$ Núcleo de Pesquisas Epidemiológicas em Nutrição e Saúde, Universidade de São Paulo (Nupens/USP) São Paulo, SP, Brasil ${ }^{3}$ Universidade Federal do Pará (UFPA), Belém, PA, Brasil
Correspondência para: Maria Alice Altenburg de Assis Centro de Ciências da Saúde, UFSC Campus Universitário, Trindade. 88040-900 - Florianópolis, SC, Brasil

massis@ccs.ufsc.br

Recebido em 10/Nov/2008 Aceito em 1/Set/2009

\section{RESUMO}

Objetivo: Investigar fatores sociodemográficos, de risco ou de proteção para doenças crônicas não transmissíveis (DCNT) que se associem ao aumento do índice de massa corporal (IMC) após os 20 anos de idade. Métodos: Estudo transversal com 769 mulheres e 572 homens do Sistema Municipal de Monitoramento de Fatores de Risco para DCNT, 2005, Florianópolis, SC. $O$ aumento do IMC foi definido em percentagem, pela diferença entre o IMC em 2005 e aos 20 anos. Resultados: Desde os 20 anos, o aumento do IMC foi superior a 10\% para a maioria dos indivíduos. Nas análises múltiplas, o aumento do IMC foi associado a aumento da idade, baixo nível educacional (mulheres), ser casado (homens), não trabalhar, baixo nível de percepção de saúde, pressão alta, colesterol/triglicerídeos elevados (homens), realização de dieta, sedentarismo e ex-tabagismo (mulheres). Conclusões: Estratégias de saúde para prevenir o ganho de peso em nível populacional devem considerar principalmente os fatores sociodemográficos. Arq Bras Endocrinol Metab. 2009;53(9):1146-56

\section{Descritores}

Índice de massa corporal; monitoramento do estado de saúde; entrevistas; fatores de risco; doenças crônicas não transmissíveis; obesidade

\begin{abstract}
Objective: To examine sociodemographic risk or protection factors for chronic non-communicable diseases (CNCDs) that may be associated with increase in body mass index (BMI) after the age of 20. Methods: Cross-sectional analysis based on data from 769 women and 572 men who participated in the 2005 Surveillance System for Risk factors for CNCDs, Florianópolis, Brazil. BMI increase was defined in percentage as the difference between BMI in 2005 and at age 20. Results: Since the age of 20 , most of the respondents had increased their BMI by more than $10 \%$. In multiples analysis, independent correlates of BMI increase were: advancing age, low education (women), being married (men), not working, low self-rated health, high blood pressure, high cholesterol/triglyceride levels (men), going on a diet, sedentarism and having been a smoker (women). Conclusions: Health promotion strategies to prevent weight gain need to be targeted to groups and should mainly consider sociodemographic factors. Arq Bras Endocrinol Metab. 2009;53(9):1146-56

Keywords:

Body mass index; health status monitoring; interviews; risk factors; chronic non-communicable diseases; obesity
\end{abstract}

\section{INTRODUÇÃO}

A s prevalências de excesso de peso e obesidade auAmentaram substancialmente nas últimas décadas
$(1,2)$. Segundo a Pesquisa de Orçamento Familiar (POF) de 2002-2003 (2), cerca de $40 \%$ da população adulta brasileira apresentava excesso de peso e, entre 
estes, $8,9 \%$ de homens e $13,1 \%$ de mulheres eram obesos. Comparando-se estimativas da POF de 2002-2003 com as de pesquisas anteriores realizadas no Brasil em 1974 e 1975 (Estudo Nacional da Despesa Familiar, ENDEF) e em 1989 (Pesquisa Nacional sobre Saúde e Nutrição, PNSN), entre os homens, a prevalência do excesso de peso duplicou e a de obesidade triplicou. A evolução de excesso de peso e obesidade entre mulheres mostrou aumentos de cerca de 50\% entre 1974 e 1989 e relativa estabilidade entre 1989 e 2003 (2).

O sobrepeso e as flutuações de ganho de peso na vida adulta estão relacionados ao aumento do risco de mortalidade e de desenvolvimento de doenças crônicas não transmissíveis (DCNTs) (1). Um elevado ganho de peso na vida adulta tem sido associado ao aumento da incidência de câncer de mama, doença cardiovascular na meia-idade e em fases mais tardias da vida, e à síndrome metabólica $(3,4)$. A Organização Mundial da Saúde (OMS) recomenda, para indivíduos adultos, uma faixa de índice de massa corporal (IMC) de 18,5 a $24,9 \mathrm{~kg} / \mathrm{m}^{2}$ e sugere, ainda, que se evitem ganhos de peso maiores do que $5 \mathrm{~kg}$ ao longo da vida adulta (3).

Diversos fatores biológicos, demográficos, socioculturais e comportamentais podem estar associados ao ganho de peso. Fatores genéticos, idade, excesso de peso no início da vida adulta, nível socioeconômico e de escolaridade, paridade, sedentarismo e etnia estão entre os fatores associados à mudança de peso no curso da vida $(5,6)$. Uma revisão realizada por Monteiro e cols. (7), reunindo estudos publicados entre 1989 e 2003 que avaliaram associações entre nível socioeconômico e obesidade em populações adultas de países em desenvolvimento, mostrou uma associação positiva para os homens, mas para as mulheres, uma associação inversa.

No Brasil, são escassos os estudos sobre mudança de peso $(6,8)$ nas diversas fases da vida adulta, bem como a sua associação com fatores sociodemográficos e fatores de risco ou proteção para DCNTs.

No presente estudo, foi analisado o aumento do IMC após os 20 anos de idade e sua associação com os fatores sociodemográficos e indicadores de risco e de proteção para DCNTs em adultos da cidade de Florianópolis.

\section{MÉTODOS}

Este é um estudo transversal que abrange adultos com idade igual ou superior a 21 anos, residentes em domicílios servidos por linhas telefônicas fixas no município de Florianópolis. Trata-se de uma amostra dos parti- cipantes do Sistema Municipal de Monitoramento de Fatores de Risco para Doenças Crônicas Não Transmissíveis, a partir de Entrevistas Telefônicas (Simtel), cujos dados foram coletados em amostra probabilística da população adulta ( $\geq 18$ anos) residente em domicílios servidos por linhas telefônicas fixas, no ano de 2005, em Florianópolis, e em outras quatro capitais brasileiras (Belém, Goiânia, Salvador e São Paulo). Foi estipulado um número mínimo de duas mil entrevistas, permitindo estimar com nível de confiança de $95 \%$ e erro máximo de dois pontos percentuais a frequência de qualquer fator de risco na população estudada (9). Outras publicações detalharam a fundamentação científica e os métodos do Simtel (9), e o plano amostral desse sistema em Florianópolis (10).

Em Florianópolis foram realizadas 2.013 entrevistas (809 homens e 1.204 mulheres) entre maio e dezembro de 2005. Foram consideradas elegíveis para o sistema 3.280 linhas. As linhas não elegíveis foram aquelas fora de serviço, as que pertenciam a empresas ou que não existiam mais $(\mathrm{n}=1.970)$. As perdas corresponderam às ligações que não responderam a dez chamadas realizadas em dias e horários distintos, linhas com sinal de ocupado, fax ou secretária eletrônica, as concluídas para residências em que não se conseguiu contato com algum morador adulto para aquiescência e sorteio, bem como quando se realizava uma ligação para residências e, não obstante a realização do sorteio, não era possível novo contato para a entrevista (seguindo o mesmo procedimento das dez chamadas) $(\mathrm{n}=963)$. A taxa final de sucesso do estudo (número de entrevistas/número de linhas elegíveis) foi de $61,4 \%$; as perdas representaram $29,4 \%$ e as recusas, $9,3 \%$. As entrevistas tiveram a duração média de 7,5 (desvio-padrão $=3,3$ ) minutos. Visando ao controle posterior de qualidade, todas as 500 entrevistas iniciais e uma amostra aleatória de $20 \%$ das entrevistas subsequentes foram revisadas e, quando necessário, realizou-se novo contato telefônico com o entrevistado para checagem de respostas.

Para as análises do presente estudo, foram excluídos os dados dos indivíduos com menos de 21 anos de idade $(\mathrm{n}=123)$, das gestantes $(\mathrm{n}=16)$, e dos indivíduos que não informaram o peso atual $(\mathrm{n}=28)$, a altura atual $(\mathrm{n}=65)$, o peso aos 20 anos de idade $(\mathrm{n}=272)$ e aqueles que apresentaram perda de peso após os 21 anos de idade $(\mathrm{n}=168)$.

O questionário do Simtel era constituído por 75 perguntas curtas e simples que abordavam: a) características demográficas e socioeconômicas; b) características 
do padrão de alimentação e de atividade física associadas à ocorrência de DCNTs; c) frequência do consumo de cigarros e de bebidas alcoólicas; e d) autoavaliação do estado de saúde e referência a diagnóstico médico anterior de hipertensão arterial, colesterol e triglicérides elevados, diabetes e osteoporose. O questionário foi submetido a estudos de validação e reprodutibilidade para os indicadores de atividade física e sedentarismo (11) e do consumo de alimentos e bebidas alcoólicas (12) com os participantes do Simtel/São Paulo. A avaliação indicou boa reprodutibilidade e adequada validade para a maioria desses indicadores $(11,12)$.

A variável de desfecho desse estudo foi o aumento relativo do IMC após os 20 anos de idade. O peso e a altura atual (em 2005) e o peso aos 20 anos de idade foram autorreferidos e utilizados para computar o IMC aos 20 anos e o IMC atual (peso em $\mathrm{kg}$ dividido pelo quadrado da altura em metros $-\mathrm{kg} / \mathrm{m}^{2}$ ). O aumento relativo do IMC foi computado pela diferença entre o IMC atual e o IMC aos 20 anos de idade dividida pelo IMC aos 20 anos de idade, multiplicado por 100.

Os indicadores selecionados do questionário Simtel para estudar a associação com o aumento do IMC após os 20 anos de idade envolveram variáveis sociodemográficas (idade, peso e altura, cor da pele, escolaridade, estado civil e trabalho remunerado); de saúde (estado de saúde autorreferido, excesso de peso aos 20 anos de idade, diagnóstico médico anterior de pressão alta e de colesterol ou triglicerídeo elevado); indicadores de risco (dieta no último ano, sedentarismo, tabagismo, consumo abusivo de bebida alcoólica, consumo habitual de refrigerantes com ou sem açúcar, hábito de consumo de leite integral e de carnes gordurosas) e de proteção (consumo de frutas, legumes e verduras [FLV]), consumo de feijão) para DCNTs.

A idade referida em anos completos foi agrupada em faixa etária (21-30, 31-40, 41-50, e 51 e mais anos). A cor da pele, referida por meio das opções branca, negra, parda ou morena e amarela, foi classificada em "branca" ou "não branca". A escolaridade foi separada em três grupos por anos de estudo $(\leq 8,9-11$, ou $\geq 12$ anos). O estado civil (opções solteiro, casado, viúvo/ separado) foi classificado em "casado" ou "não casado". A existência de trabalho remunerado foi indicada por meio de respostas "sim" ou "não".

O estado de saúde autorreferido pelo entrevistado como excelente, bom, regular ou ruim foi agrupado como "excelente/bom" ou "regular/ruim". Resposta "não" ou "sim" identificava a referência ao diagnósti- co médico anterior de pressão alta, e de colesterol ou triglicerídeo elevado. Na variável excesso de peso aos 20 anos (não/sim), foi considerado IMC $\geq 25 \mathrm{~kg} / \mathrm{m}^{2}$ (l). Finalmente, classificaram-se os indivíduos segundo categorias de aumento relativo do IMC após os 20 anos de idade em: 0 a $9 \%, 10 \%$ a $19 \%, 20 \%$ a $29 \%$ ou $\geq 30 \%$.

Entre os indicadores comportamentais, foram incluídas as variáveis: realização de dieta para perda de peso no último ano (não/sim), sedentarismo (não/sim), tabagismo (atual, nunca ou ex-fumante). O consumo abusivo de bebida alcoólica foi obtido pela resposta "não" ou "sim" à pergunta sobre a ingestão de cinco doses de qualquer bebida alcoólica em pelo menos um dia do último mês, independentemente do sexo. Foram considerados sedentários (não/sim) os indivíduos que concomitantemente referiram a) não realizar atividade física por lazer; b) realizar esforço físico leve ou muito leve no trabalho; c) não se deslocar para o trabalho a pé ou de bicicleta; d) não realizar sozinhos a limpeza pesada de suas casas.

Os indicadores de risco e proteção dietéticos para DCNTs foram formados por variáveis marcadoras de consumo alimentar não saudável (consumo de refrigerantes com ou sem açúcar em uma ou mais vezes na semana [não/sim], consumo de leite integral [não/sim] e consumo de carne com gordura ou frango com pele [não/sim]) e saudável (consumo de FLV em cinco ou mais vezes por semana [sim/não], consumo de feijão com frequência igual ou maior a três vezes por semana [ $\operatorname{sim} /$ não]). A escolha desses indicadores foi baseada nas recomendações do Guia Alimentar da população brasileira (13) e da Análise da Estratégia Global para Alimentação Saudável, Atividade Física e Saúde (14).

Para verificação da associação entre o aumento relativo de IMC após os 20 anos de idade e as variáveis independentes, foi realizado teste de diferenças de médias de $t$ de Student e análise de regressão linear simples e múltipla, considerando-se o intervalo de confiança de 95\% e nível de significância de 5\%. Para a análise de regressão linear múltipla, estruturou-se um marco teórico com as variáveis independentes selecionadas a partir dos resultados presentes na literatura $(5,15,16)$ e disponíveis no banco de dados, discriminadas em blocos hierarquizados e ordenadas de acordo com a procedência com que atuariam sobre o desfecho (17). Os blocos apresentaram a seguinte ordem: características sociodemográficas, de saúde, comportamentais e consumo alimentar. Com exceção do IMC aos 20 anos de idade, as demais variáveis independentes eram categóricas 
e, para estas, os coeficientes de regressão apresentam a diferença do aumento relativo do IMC de uma categoria específica comparada à categoria de referência. As variáveis com $\mathrm{p}<0,20$ foram introduzidas no modelo conforme o nível hierárquico, para controle de fatores de confusão. Consideraram-se fatores associados ao aumento do IMC aqueles com teste de heterogeneidade ou de tendência linear significativo $(\mathrm{p}<0,05)$. As análises foram estratificadas por sexo e realizadas no programa STATA 9.0 (Statistical Analysis System software, Release 9, 2005).

Em se tratando de entrevista por telefone, o termo de consentimento livre e esclarecido foi substituído pelo consentimento verbal obtido por ocasião dos contatos telefônicos com os entrevistados. O projeto de pesquisa foi aprovado primeiramente pelo Comitê de Ética em Pesquisa da Universidade de São Paulo (USP) e posteriormente pelo Comitê de Ética em Pesquisa da Universidade Federal de Santa Catarina (protocolo $n^{\circ}$. 077/05).

\section{RESULTADOS}

A amostra deste estudo foi composta por 1.341 pessoas, 769 mulheres e 572 homens. A idade média (desvio-padrão) dos entrevistados foi de 40,6 anos $(13,4)$ para as mulheres e de 40,6 (13,8) anos para os homens. As mulheres apresentaram em média 11,9 anos de estudo $(4,2)$ e os homens, 12 anos de estudo $(4,2)$.

A tabela 1 apresenta os dados de peso, de IMC, o percentual de excesso de peso aos 20 anos e na entrevista (2005) e frequência de indivíduos, segundo categorias de aumento do IMC, após os 20 anos de idade. A maioria dos homens e das mulheres ganhou mais do que $10 \%$ do seu IMC inicial; um quarto das mulheres e $14 \%$ dos homens apresentaram um aumento do IMC maior do que $30 \%$. A frequência de excesso de peso no ano da entrevista (2005) foi maior para os homens do que para as mulheres, mas o percentual de aumento do IMC (média \pm DP) após os 20 anos de idade foi maior para as mulheres $(20,5 \% \pm 19,4 \%)$ do que para os homens $(16,8 \% \pm 14,4 \%), \mathrm{p}<0,001$. Entre os 20 anos de idade e a idade referida em 2005, a média do IMC aumentou de $20,2( \pm 2,4) \mathrm{kg} / \mathrm{m}^{2}$ para $24,2( \pm 4,2 \%)$ $\mathrm{kg} / \mathrm{m}^{2}$ entre as mulheres e de $22,1( \pm 2,5) \mathrm{kg} / \mathrm{m}^{2}$ para $25,7( \pm 3,7) \mathrm{kg} / \mathrm{m}^{2}$ entre os homens. Isso equivale a um aumento médio de $4,0( \pm 3,6) \mathrm{kg} / \mathrm{m}^{2}$ ou $10,4 \mathrm{~kg}$ $( \pm 9,3)$ para as mulheres e de $3,6( \pm 2,9) \mathrm{kg} / \mathrm{m}^{2}$ ou $11,1 \mathrm{~kg}( \pm 9,1)$ para os homens ( $\mathrm{p}=0,03$ para aumento de IMC). Em média, o aumento anual de IMC foi de 0,3 unidade, para ambos os sexos, o que equivale a um ganho de peso médio de $0,7 \mathrm{~kg}( \pm 0,9 \mathrm{~kg})$ por ano (homens $=0,9 \pm 1,2 \mathrm{~kg}$ /ano; mulheres $=0,7 \pm 0,7 \mathrm{~kg} /$ ano, $\mathrm{p}<0,001)$.

As tabelas 2 e 3 apresentam as médias e os desviospadrões do aumento relativo do IMC após os 20 anos de idade e as análises de associação bivariadas com as variáveis independentes para as mulheres e os homens, respectivamente.

Entre as mulheres, nas análises bivariadas, o aumento de IMC foi associado às categorias de faixa etária igual ou superior a 31 anos, nível de escolaridade inferior a 12 anos, ao fato de não trabalharem, à referência ao estado de saúde como regular ou ruim, ao diagnóstico médico de pressão alta e de colesterol/triglicerídeos elevados, à realização de dieta para perda de peso no último ano, ao sedentarismo, não tabagismo e ex-tabagismo. As variáveis IMC aos 20 anos e consumo abusivo de bebida alcoólica apresentaram associação inversa ao aumento do IMC (Tabela 2).

Entre os homens, nas análises bivariadas, o aumento do IMC foi associado às categorias de faixa etária igual ou superior a 31 anos, aos casados e ao fato de não trabalharem, à referência ao estado de saúde como regular ou ruim, ao diagnóstico médico anterior de pressão alta e de colesterol/triglicerídeos elevados, à realização de dieta no último ano, ao sedentarismo e ao ex-tabagismo. O IMC aos 20 anos de idade apresentou uma associação inversa ao aumento do IMC (Tabela 3 ).

As análises múltiplas hierarquizadas confirmaram a maioria das associações identificadas nas análises bivariadas para as mulheres, mas anularam as associações com o diagnóstico médico anterior de colesterol/triglicerídeos elevados e com o consumo abusivo de bebida alcoólica. A análise múltipla também modificou a magnitude de efeito do consumo de carne ou frango com gordura, resultando numa associação limítrofe entre o aumento do IMC após os 20 anos e esta variável $(\mathrm{p}=$ $0,06)$. Assim, entre as mulheres, o aumento de IMC após os 20 anos de idade foi associado às categorias de faixa etária igual ou superior a 31 anos, de nível de escolaridade inferior a 12 anos, ao fato de não trabalharem, à referência ao estado de saúde como regular ou ruim, ao diagnóstico médico de pressão alta, ao IMC aos 20 anos, à realização de dieta no último ano, ao sedentarismo e hábito de fumar (nunca e ex-fumante). As variáveis do modelo final das análises múltiplas explicaram $38,8 \%$ da variabilidade do aumento do IMC após os 20 anos (Tabela 4 ). 
Tabela 1. Peso, índice de massa corporal, frequência de excesso de peso aos 20 anos de idade e no ano de 2005 e frequência de indivíduos, segundo categorias de aumento do IMC, após os 20 anos de idade, em mulheres e homens (Simtel/Florianópolis, 2005)

\begin{tabular}{|c|c|c|}
\hline Variáveis & Mulheres $(n=769)$ & Homens $(n=572)$ \\
\hline \multicolumn{3}{|l|}{ Aos 20 anos } \\
\hline Peso (kg) (média [DP]) & $52,8(7,3)$ & $67,7(9,0)$ \\
\hline IMC (kg/m²) (média [DP]) & $20,2(2,4)$ & $22,1(2,5)$ \\
\hline Excesso de peso ${ }^{1}(\%)$ & $3,8(2,4-5,1)$ & $11,2(8,6-13,8)$ \\
\hline \multicolumn{3}{|l|}{ Ano de 2005} \\
\hline Peso (kg) (média [DP]) & $63,2(10,9)$ & $78,8(12,4)$ \\
\hline IMC (kg/m²) (média [DP]) & $24,2(4,2)$ & $25,7(3,7)$ \\
\hline Excesso de peso ${ }^{1}(\%)$ & $34,9(31,5-38,2)$ & $53,1(49,0-57,2)$ \\
\hline \multicolumn{3}{|c|}{ Percentual de aumento do IMC após os 20 anos de idade (\%) } \\
\hline $0-9$ & $37,7(34,3-41,1)$ & $36,2(32,2-40,1)$ \\
\hline $10-19$ & $20,9(18,1-23,8)$ & $30,2(26,5-34,0)$ \\
\hline $20-29$ & $16,1(13,5-18,7)$ & $19,6(16,3-22,8)$ \\
\hline $30+$ & $25,2(22,2-28,3)$ & $14,0(11,1-16,8)$ \\
\hline
\end{tabular}

${ }^{1}\left(\mathrm{IMC} \geq 25 \mathrm{~kg} / \mathrm{m}^{2}\right)$.

DP: desvio-padrão; IC95\%: intervalo de confiança de $95 \%$.

Tabela 2. Percentual de aumento do índice de massa corporal (média e desvio-padrão) após os 20 anos e associações bivariadas com variáveis independentes para as mulheres (Simtel/Florianópolis, 2005)

\begin{tabular}{|c|c|c|c|c|c|c|}
\hline \multirow{2}{*}{ Variáveis } & \multirow{2}{*}{$\mathbf{N}$} & \multirow{2}{*}{ Média } & \multirow{2}{*}{ DP } & \multicolumn{3}{|c|}{ Análises bivariadas } \\
\hline & & & & $\beta$ & IC 95\% & $\mathbf{p}$ \\
\hline \multicolumn{7}{|l|}{ Sociodemográficas } \\
\hline Faixa etária (anos) & & & & & & 0,000 \\
\hline $21-30$ & 212 & 9,1 & 9,3 & & & \\
\hline $31-40$ & 204 & 18,0 & 16,4 & 8,95 & 5,$60 ; 12,30$ & \\
\hline $41-50$ & 181 & 26,3 & 22,0 & 17,24 & 13,$78 ; 20,69$ & \\
\hline $51+$ & 172 & 31,6 & 20,5 & 22,53 & 19,$03 ; 26,03$ & \\
\hline Cor da pele & & & & & & 0,081 \\
\hline Não branca & 197 & 22,6 & 20,1 & & & \\
\hline Branca & 572 & 19,8 & 19,1 & $-2,79$ & $-5,92 ; 0,35$ & \\
\hline Anos de estudo & & & & & & 0,000 \\
\hline$\geq 12$ & 354 & 16,9 & 17,6 & & & \\
\hline $9-11$ & 253 & 21,0 & 19,0 & 4,12 & 1,$06 ; 7,18$ & \\
\hline$\leq 8$ & 162 & 27,9 & 21,5 & 11,0 & 7,$48 ; 14,53$ & \\
\hline Estado civil (casada) & & & & & & 0,203 \\
\hline Não & 360 & 19,6 & 19,2 & & & \\
\hline Sim & 409 & 21,4 & 19,5 & 1,78 & $-0,96 ; 4,53$ & \\
\hline Trabalho & & & & & & 0,000 \\
\hline Sim & 485 & 17,2 & 16,7 & & & \\
\hline Não & 284 & 26,2 & 22,1 & 8,94 & 6,$17 ; 11,71$ & \\
\hline \multicolumn{7}{|l|}{ Saúde } \\
\hline Estado de saúde & & & & & & 0,000 \\
\hline Excelente/bom & 558 & 17,1 & 16,7 & & & \\
\hline Regular/ruim & 211 & 29,6 & 22,7 & 12,44 & 9,$49 ; 15,38$ & \\
\hline Pressão alta & & & & & & 0,000 \\
\hline Não & 636 & 17,5 & 16,8 & & & \\
\hline Sim & 133 & 35,3 & 23,6 & 17,84 & 14,$44 ; 21,24$ & \\
\hline Colesterol/triglicérides elevados & & & & & & 0,000 \\
\hline Não & 622 & 18,7 & 18,5 & & & \\
\hline Sim & 147 & 28,2 & 21,1 & 9,41 & 5,$99 ; 12,84$ & \\
\hline IMC 20 anos & 769 & 20,5 & 19,4 & $-1,83$ & $-2,37 ;-1,28$ & \\
\hline
\end{tabular}




\begin{tabular}{|c|c|c|c|c|c|c|}
\hline Tabela 2. Continuação & & & & & & \\
\hline Comportamentais & & & & & & \\
\hline Dieta para perda de peso & & & & & & 0,000 \\
\hline Não & 480 & 17,9 & 18,4 & & & \\
\hline $\operatorname{Sim}$ & 289 & 24,9 & 20,1 & 6,94 & 4,$16 ; 9,73$ & \\
\hline Sedentarismo & & & & & & 0,000 \\
\hline Não & 626 & 19,0 & 17,4 & & & \\
\hline Sim & 143 & 27,1 & 25,2 & 8,06 & 4,$59 ; 11,54$ & \\
\hline Hábito de fumar & & & & & & 0,007 \\
\hline Atual & 150 & 16,9 & 15,7 & & & \\
\hline Nunca & 473 & 20,6 & 19,9 & 3,71 & 0,$17 ; 7,25$ & \\
\hline Ex-fumante & 146 & 24,0 & 20,4 & 7,08 & 2,$68 ; 11,47$ & \\
\hline Consumo abusivo de bebida alcoólica ${ }^{\mathrm{a}}$ & & & & & & 0,001 \\
\hline Não & 691 & 21,3 & 19,9 & & & \\
\hline Sim & 78 & 13,8 & 11,1 & $-7,49$ & $-12,00 ;-2,98$ & \\
\hline Consumo alimentar & & & & & & \\
\hline Consumo regular de FLV (5/sem & & & & & & 0,167 \\
\hline Sim & 573 & 21,1 & 19,6 & & & \\
\hline Não & 196 & 18,9 & 18,5 & $-2,22$ & $-5,36 ; 0,93$ & \\
\hline Consumo de feijão (dias/semana) & & & & & & 0,555 \\
\hline$\geq 3$ & 383 & 20,1 & 18,9 & & & \\
\hline$<3$ & 386 & 21,0 & 19,8 & 0,82 & $-1,92 ; 3,57$ & \\
\hline $\begin{array}{l}\text { Consumo de carne ou frango com } \\
\text { gordura }\end{array}$ & & & & & & 0,154 \\
\hline Não & 595 & 20,0 & 19,3 & & & \\
\hline $\operatorname{Sim}$ & 174 & 22,4 & 19,7 & 2,38 & $-0,89 ; 5,65$ & \\
\hline Consumo de leite integral & & & & & & 0,640 \\
\hline Não & 453 & 20,8 & 19,3 & & & \\
\hline $\operatorname{Sim}$ & 316 & 20,2 & 19,5 & $-0,66$ & $-3,45 ; 2,12$ & \\
\hline Hábito de tomar refrigerante & & & & & & 0,767 \\
\hline Não & 305 & 20,3 & 19,3 & & & \\
\hline Sim & 464 & 20,7 & 19,4 & 0,42 & $-2,38 ; 3,23$ & \\
\hline
\end{tabular}

a $\geq 5$ doses/dia pelo menos 1 vez no último mês.

FLV: frutas, legumes e verduras; IC 95\%: intervalo de confiança de $95 \%$.

\begin{tabular}{|c|c|c|c|c|c|c|}
\hline \multirow{2}{*}{ Variáveis } & \multirow{2}{*}{$\mathbf{N}$} & \multirow{2}{*}{ Média } & \multirow{2}{*}{ DP } & \multicolumn{3}{|c|}{ Análises bivariadas } \\
\hline & & & & $\beta$ & IC 95\% & p \\
\hline \multicolumn{7}{|l|}{ Sociodemográficas } \\
\hline Faixa etária (anos) & & & & & & 0,000 \\
\hline $21-30$ & 152 & 11,4 & 9,8 & & & \\
\hline $31-40$ & 174 & 16,1 & 11,9 & 4,74 & 1,$72 ; 7,77$ & \\
\hline $41-50$ & 116 & 18,6 & 13,4 & 7,20 & 3,$84 ; 10,56$ & \\
\hline $51+$ & 130 & 22,6 & 19,7 & 11,21 & 7,$96 ; 14,47$ & \\
\hline Cor da pele & & & & & & 0,557 \\
\hline Não branca & 167 & 16,3 & 12,3 & & & \\
\hline Branca & 405 & 17,0 & 15,2 & 0,78 & $-1,83 ; 3,38$ & \\
\hline Anos de estudo & & & & & & 0,185 \\
\hline$\geq 12$ & 277 & 16,2 & 12,6 & & & \\
\hline $9-11$ & 175 & 16,7 & 13,9 & 0,45 & $-2,28 ; 3,19$ & \\
\hline$\leq 8$ & 120 & 18,4 & 18,6 & 2,21 & $-0,88 ; 5,30$ & \\
\hline Estado civil (casado) & & & & & & 0,000 \\
\hline Não & 196 & 12,1 & 10,8 & & & \\
\hline $\operatorname{Sim}$ & 376 & 19,3 & 15,4 & 7,23 & 4,$81 ; 9,66$ & \\
\hline
\end{tabular}




\begin{tabular}{|c|c|c|c|c|c|c|}
\hline \multicolumn{7}{|l|}{ Tabela 3. Continuação } \\
\hline Trabalho & & & & & & 0,011 \\
\hline $\operatorname{Sim}$ & 445 & 16,0 & 12,4 & & & \\
\hline Não & 127 & 19,7 & 19,7 & 3,66 & 0,$83 ; 6,49$ & \\
\hline \multicolumn{7}{|l|}{ Saúde } \\
\hline Estado de saúde & & & & & & 0,002 \\
\hline Excelente/bom & 478 & 16,0 & 13,5 & & & \\
\hline Regular/ruim & 94 & 21,0 & 18,0 & 4,96 & 1,$79 ; 8,13$ & \\
\hline Pressão alta & & & & & & 0,000 \\
\hline Não & 471 & 15,3 & 12,4 & & & \\
\hline Sim & 101 & 23,8 & 20,1 & 8,51 & 5,$48 ; 11,54$ & \\
\hline Colesterol/triglicérides elevados & & & & & & 0,000 \\
\hline Não & 480 & 15,5 & 13,0 & & & \\
\hline $\operatorname{Sim}$ & 92 & 23,7 & 19,0 & 8,20 & 5,$05 ; 11,36$ & \\
\hline IMC 20 anos & 572 & 16,8 & 14,4 & $-1,40$ & $-1,86 ;-0,94$ & 0,000 \\
\hline \multicolumn{7}{|l|}{ Comportamentais } \\
\hline Dieta para perda de peso & & & & & & 0,000 \\
\hline Não & 469 & 15,4 & 13,8 & & & \\
\hline Sim & 103 & 23,1 & 15,4 & 7,62 & 4,$61 ; 10,64$ & \\
\hline Sedentarismo & & & & & & 0,002 \\
\hline Não & 429 & 15,8 & 12,9 & & & \\
\hline $\operatorname{Sim}$ & 143 & 20,0 & 17,8 & 4,24 & 1,$53 ; 6,96$ & \\
\hline Hábito de fumar & & & & & & 0,015 \\
\hline Atual & 118 & 15,0 & 12,1 & & & \\
\hline Nunca & 297 & 16,1 & 12,7 & 1,15 & $-1,91 ; 4,22$ & \\
\hline Ex-fumante & 157 & 19,6 & 18,3 & 4,59 & 1,16; 8,02 & \\
\hline Consumo abusivo de bebida alcoólica ${ }^{a}$ & & & & & & 0,187 \\
\hline Não & 384 & 17,4 & 15,7 & & & \\
\hline $\operatorname{Sim}$ & 188 & 15,7 & 11,3 & $-1,69$ & $-4,21 ; 0,82$ & \\
\hline Consumo alimentar & & & & & & 0,579 \\
\hline \multicolumn{7}{|l|}{ Consumo regular de FLV (5/sem) } \\
\hline Não & 379 & 16,6 & 14,8 & & & \\
\hline Sim & 193 & 17,3 & 13,6 & 0,71 & $-1,80 ; 3,21$ & \\
\hline Consumo de feijão (dias/semana) & & & & & & 0,079 \\
\hline$\geq 3$ & 374 & 16,0 & 13,7 & & & \\
\hline$<3$ & 198 & 18,3 & 15,5 & 2,23 & $-0,26 ; 4,71$ & \\
\hline $\begin{array}{l}\text { Consumo de carne ou frango com } \\
\text { gordura }\end{array}$ & & & & & & 0,465 \\
\hline Não & 332 & 17,2 & 14,2 & & & \\
\hline $\operatorname{Sim}$ & 240 & 16,3 & 14,7 & $-0,89$ & $-3,29 ; 1,51$ & \\
\hline Consumo de leite integral & & & & & & 0,689 \\
\hline Não & 297 & 17,0 & 14,5 & & & \\
\hline $\operatorname{Sim}$ & 275 & 16,6 & 14,4 & $-0,48$ & $-2,85 ; 1,89$ & \\
\hline Hábito de tomar refrigerante & & & & & & 0,399 \\
\hline Não & 216 & 17,5 & 15,8 & & & \\
\hline $\operatorname{Sim}$ & 356 & 16,4 & 13,5 & $-1,05$ & $-3,49 ; 1,39$ & \\
\hline
\end{tabular}

a $\geq 5$ doses/dia pelo menos 1 vez no último mês.

FLV: frutas, legumes e verduras; IC95\%: intervalo de confiança de $95 \%$.

Nas análises múltiplas, para os homens, a categoria de faixa etária entre 31 e 40 anos e as variáveis de autoavaliação do estado de saúde e hábito de fumar perderam significância após os ajustes. Desse modo, entre os ho- mens, o aumento de IMC após os 20 anos de idade foi associado às categorias de faixa etária igual ou superior a 41 anos, ao fato de serem casados e não trabalharem, ao diagnóstico médico de pressão alta e de colesterol/ 
triglicerídeos elevados, ao IMC aos 20 anos, à realização de dieta para perda de peso no último ano. Para o sedentarismo, observou-se uma significância limítrofe $(\mathrm{p}=0,055)$. O modelo obtido para os homens apresentou $24,6 \%$ de capacidade preditiva da variabilidade total do aumento do IMC após os 20 anos (Tabela 4).

\section{DISCUSSÃO}

O nível de aumento do IMC e de ganho de peso observado nesta amostra de adultos de Florianópolis foi similar aos dados de estudos que investigaram o ganho de peso na meia-idade, com informações autorreferidas de peso aos 18 (18) e aos 20 anos (5). O aumento médio do IMC após os 20 anos de idade foi significativamente maior para as mulheres do que para os homens $(20,5 \%$ versus $16,8 \%$ ), consistente com achados de estudos longitudinais que investigaram a mudança do IMC num período de seis anos a partir dos 20 anos de idade (19) e de estudos transversais com dados do Behavioral Risk Factor Surveillance System (BRFSS) nos Estados Uni$\operatorname{dos}(20)$.

Considera-se um ponto positivo deste estudo as análises múltiplas e hierarquizadas para avaliar as associações do aumento do IMC (\%) após os 20 anos de idade com os fatores sociodemográficos, de saúde, comportamentais e de consumo alimentar. O estudo também apresenta inovação na problemática da obesidade para a Saúde Pública, focando os fatores associados ao aumento do IMC em vez da frequência de excesso de peso.

Este estudo apresenta algumas limitações. Primeiro, o padrão de aumento de IMC verificado refere-se a uma amostra de adultos do Simtel/Florianópolis e não pode ser generalizado para a população do município tampouco para a população brasileira em geral. A amostra entrevistada pelo Simtel só permite inferências populacionais para a população adulta que reside em domicílios cobertos pela rede de telefonia fixa, que é distinta nas várias capitais do Brasil e não é universal, podendo ser baixa nos estratos de menor nível socioeconômico. Para atender aos objetivos deste estudo, foi excluído um terço dos entrevistados, entre eles, os que não informaram a altura atual, o peso atual e aos 20 anos de idade. Em vista disso, não foram aplicados os pesos pós-estratificação, recomendados para corrigir, ao menos parcialmente, vieses determinados pela não cobertura universal da rede telefônica (9). Em segundo lugar, inquéritos realizados por telefone não garantem que o sujeito sorteado tenha sido o respondente. Ter- ceiro, os dados autorrelatados podem estar sujeitos a vieses decorrentes da memória ou de comportamentos socialmente desejáveis. Tem sido frequente a utilização de peso e altura autorreferidos em estudos epidemiológicos, para avaliar a associação do ganho de peso com fatores sociodemográficos e de estilo de vida $(16,20,21)$. Estudos realizados no Brasil mostraram que as medidas de concordância entre peso e altura referidos e aferidos foram bastante altas $(6,21)$. O peso corporal referido é frequentemente subestimado e a altura, superestimada em relação às medidas aferidas (21). Existe, pois, a possibilidade de subestimativas do aumento do IMC, na amostra do presente estudo, e da prevalência do excesso de peso, na população total investigada pelo Simtel/ Florianópolis $(\mathrm{n}=2.013)$. No entanto, a prevalência de excesso de peso para a população adulta com telefone no Simtel/Florianópolis em 2005 (45,2\% entre os homens e $33,7 \%$ entre as mulheres [dados não apresentados]) foi similar à encontrada pelo sistema Vigitel (Vigilância de Fatores de Risco e Proteção para Doenças Crônicas por Inquérito Telefônico) no ano de 2006 em Florianópolis $(48,5 \%$ entre os homens e de $32,2 \%$ entre as mulheres) (22). Futuros estudos devem ser conduzidos para avaliar a validade de peso e altura referidos contra as informações aferidas para que as medidas obtidas pelo sistema Vigitel sejam corrigidas.

Finalmente, destaca-se a limitação referente à natureza transversal do estudo: faltam informações sobre as flutuações de peso e de IMC entre a idade de 20 anos e a data da coleta dos dados. Estudos mostraram que as flutuações de peso e de IMC ao longo da vida adulta foram associadas a alguns desfechos, tais como a síndrome metabólica (4).

O presente estudo evidenciou que os fatores sociodemográficos (bloco distal) contabilizaram a maior proporção da variância total para o aumento do IMC após os 20 anos de idade em ambos os sexos (mulheres $=21,7 \%$; homens $=10,2 \%)$. Entre as variáveis sociodemográficas, o aumento da faixa etária apresentou a maior magnitude de efeito em ambos os sexos, seguido da menor escolaridade (entre as mulheres), da condição de ser casado (entre os homens) e de não trabalhar (em ambos os sexos). Fatores sociodemográficos descritos como preditores e determinantes do ganho de peso ou do aumento do IMC em estudos longitudinais conduzidos com mulheres incluíram baixo nível salarial e educacional $(5,23)$, raça negra e mulata $(6,20)$, serem casadas $(15,16)$ e aposentadas (5). Para os homens, os estudos longitudinais disponíveis para comparação produziram resultados 
Tabela 4. Análises múltiplas entre 0 aumento do índice de massa corporal e variáveis independentes para mulheres e homens (Simtel/Florianópolis, 2005)

\begin{tabular}{|c|c|c|c|c|c|c|c|c|c|c|}
\hline \multirow{3}{*}{ Variáveis } & \multicolumn{5}{|c|}{ Mulheres } & \multicolumn{5}{|c|}{ Homens } \\
\hline & \multicolumn{5}{|c|}{ Análises múltiplas } & \multicolumn{5}{|c|}{ Análises múltiplas } \\
\hline & $\beta$ & IC95\% & $\mathbf{p}^{\star}$ & $\mathbf{p}^{\star \star}$ & $\mathbf{R}^{2}$ & $\beta$ & IC95\% & $\mathbf{p}^{\star}$ & $\mathbf{p}^{\star \star}$ & $\mathbf{R}^{2}$ \\
\hline Nível $1^{\mathrm{a}}$ & & & & 0,000 & 0,217 & & & & 0,000 & 0,102 \\
\hline \multicolumn{11}{|l|}{ Faixa etária (anos) } \\
\hline $31-40$ & 8,63 & 5,$33 ; 11,93$ & & & & 3,10 & $-0,07 ; 6,26$ & & & \\
\hline $41-50$ & 16,99 & 13,$60 ; 20,39$ & & & & 5,09 & 1,$52 ; 8,66$ & & & \\
\hline $51+$ & 19,23 & 15,$54 ; 22,92$ & 0,000 & & & 7,92 & 4,$36 ; 11,48$ & 0,000 & & \\
\hline \multicolumn{11}{|l|}{ Anos de estudo } \\
\hline & 3,66 & 0,$89 ; 6,43$ & & & \multicolumn{6}{|c|}{$\geq 12$} \\
\hline$\leq 8$ & 7,01 & 3,$74 ; 10,29$ & 0,000 & & & & & & & \\
\hline \multicolumn{11}{|l|}{ Estado civil (casado) } \\
\hline $\operatorname{Sim}$ & & & & & & 5,41 & 2,$78 ; 8,04$ & 0,000 & & \\
\hline \multicolumn{11}{|l|}{ Trabalho } \\
\hline \multicolumn{11}{|l|}{$\operatorname{Sim}$} \\
\hline Não & 4,25 & 1,$52 ; 6,98$ & 0,014 & & & 3,02 & 0,$01 ; 6,02$ & 0,037 & & \\
\hline Nível 2a & & & & 0,000 & 0,309 & & & & 0,000 & 0,192 \\
\hline \multicolumn{11}{|l|}{ Estado de saúde } \\
\hline \multicolumn{11}{|l|}{ Excelente/bom } \\
\hline Regular/ruim & 7,31 & 4,$58 ; 10,04$ & 0,000 & & & & & & & \\
\hline \multicolumn{11}{|l|}{ Pressão alta } \\
\hline \multicolumn{11}{|l|}{ Não } \\
\hline $\operatorname{Sim}$ & 8,38 & 5,$05 ; 11,70$ & 0,000 & & & 5,18 & 2,$12 ; 8,25$ & 0,001 & & \\
\hline \multicolumn{11}{|c|}{ Colesterol/triglicérides elevados } \\
\hline \multicolumn{11}{|l|}{ Não } \\
\hline Sim & & & & & & 4,52 & 1,$48 ; 7,56$ & 0,004 & & \\
\hline IMC aos 20 anos & $-1,63$ & $-2,10 ;-1,15$ & 0,000 & & & $-1,41$ & $-1,84 ;-0,98$ & 0,000 & & \\
\hline Nível 3a & & & & 0,000 & 0,378 & & & & 0,000 & 0,230 \\
\hline \multicolumn{11}{|l|}{ Dieta para perda de peso } \\
\hline \multicolumn{11}{|l|}{ Não } \\
\hline Sim & 8,88 & 6,$57 ; 11,19$ & 0,000 & & & 7,43 & 4,$61 ; 10,25$ & 0,000 & & \\
\hline \multicolumn{11}{|l|}{ Sedentarismo } \\
\hline \multicolumn{11}{|l|}{ Não } \\
\hline $\operatorname{Sim}$ & 6,40 & 3,$58 ; 9,21$ & 0,000 & & & 2,39 & $-0,05 ; 4,83$ & 0,055 & & \\
\hline Hábito de fumar & & & & & & & & & & \\
\hline Atual & & & & & & & & & & \\
\hline Nunca & 3,46 & 0,$51 ; 6,42$ & & & & & & & & \\
\hline Ex-fumante & 4,86 & 1,$30 ; 8,43$ & 0,020 & & & & & & & \\
\hline Nível 4a & & & & 0,000 & 0,388 & & & & 0,000 & 0,246 \\
\hline Consumo de carne ou frang & & & & & & & & & & \\
\hline Não & & & & & & & & & & \\
\hline $\operatorname{Sim}$ & 2,48 & $-0,12 ; 5,09$ & & 0,061 & & & & & & \\
\hline
\end{tabular}

${ }^{\star}$ p variável; ** p modelo.

a Nível no modelo hierárquico.

IC95\%: intervalo de confiança de 95\%.

inconsistentes relativamente ao nível educacional, mostrando uma associação positiva (23), negativa (15) e nenhuma associação (19) com o aumento do IMC.
Relativamente às variáveis do segundo nível do modelo hierárquico (saúde), as associações encontradas no presente estudo são consistentes com as de outras publicações que avaliaram o ganho de peso e observaram 
associações com autoavaliação negativa de estado de saúde (15) e com fatores de risco cardiovascular (hipertensão, diabetes, dislipidemias) $(4,15,18)$. No bloco das variáveis de comportamentos de risco para a saúde (terceiro nível do modelo hierárquico), o aumento do IMC foi associado à realização de dieta para perda de peso em ambos os sexos, e ao sedentarismo e hábito de fumar entre as mulheres. Outros estudos observaram associações entre o ganho de peso e o sedentarismo no lazer $(5,15)$, baixo nível de atividade física no trabalho (23), não tabagismo (5), ex-tabagismo $(15,19)$ e, entre mulheres, a realização de dietas (5).

Em contraste com os achados do presente estudo, em que não foram observadas associações entre o aumento de IMC e o consumo dos alimentos investigados, estudos longitudinais sobre a relação entre ganho de peso, aumento do IMC ou circunferência da cintura mostraram associação inversa com padrões caracterizados por alto consumo de frutas, vegetais, cereais integrais e por baixo consumo de fast foods, refrigerantes e carnes processadas $(24,25)$. Existem controvérsias sobre o papel da composição dietética na determinação do desbalanceamento energético e do ganho de peso subsequente. Os problemas relacionados ao sub-relato de alimentos calóricos e super-relatos de alimentos saudáveis por indivíduos obesos são vieses de difícil controle em inquéritos populacionais. Numa revisão de trinta estudos transversais que investigaram os padrões de consumo alimentar e sua associação com o IMC e a obesidade, 11 não mostraram associações, e as associações dos demais estudos foram consideradas inconsistentes (26). No Brasil, a complexidade das relações entre padrão alimentar (PA) e obesidade foi relatada num estudo transversal de base populacional conduzido com mulheres de São Leopoldo (RS) (27). Nele, observou-se que o baixo consumo do PA-frutas foi associado positivamente ao IMC e o baixo consumo do PA-vegetais apresentou efeito protetor para o aumento nos níveis de IMC (27).

Os fatores associados ao aumento do IMC após os 20 anos observados no presente estudo sugerem que essa informação pode ser um bom marcador de outros comportamentos de risco para a saúde, especialmente entre as mulheres. As variáveis sociodemográficas e de estilo de vida não saudável descreveram um quadro incompleto dos fatores associados ao aumento do IMC, como evidenciado pelo fato de que o modelo obtido explica $38,8 \%$ da variância para as mulheres e $24,6 \%$ para os homens. Fatores sociodemográficos e de saúde não incluídos no questionário Simtel, tais como idade da menarca, paridade e terapia de reposição hormonal, têm sido associados ao aumento de peso entre mulheres $(5,16)$. Outros fatores relacionados a contextos ambientais (por exemplo, disponibilidade de lugares públicos para realizar atividades físicas de lazer) e a frequência de consumo de alimentos com alta densidade energética e gorduras saturadas (carnes gordurosas, leite integral e alimentação fora de casa) incluídos no questionário Vigitel 2007 (28) poderão explicar melhor as associações entre aumento do IMC e fatores comportamentais relacionados ao estilo de vida.

Os achados do presente estudo fornecem uma base para futuras comparações com os dados gerados pelo sistema Vigitel, podendo prover informações para um melhor entendimento dos fatores associados ao aumento do IMC na cidade de Florianópolis, foco dessa investigação.

Agradecimentos: ao Conselho Nacional de Desenvolvimento Científico e Tecnológico (CNPq Edital CT - Saúde/MCT/MS/ CNPq n ${ }^{\circ}$ 30/2004 - Alimentação e Nutrição).

Declaração: os autores declaram não haver conflitos de interesse científico neste estudo.

\section{REFERÊNCIAS}

1. World Health Organization. Obesity: Preventing and managing the global epidemic. Report of a WHO Consultation on Obesity. Geneva:WHO; 1998.

2. Instituto Brasileiro de Geografia e Estatística. Pesquisa de orçamentos familiares 2002-2003: análise da disponibilidade domiciliar de alimentos e do estado nutricional no Brasil. Rio de Janeiro: IBGE; 2004.

3. World Health Organization. Diet, nutrition and the prevention of chronic diseases. Report of a Joint WHO/FAO Expert Consultation. [WHOTechnical Report Series 916] Geneva:WHO; 2003.

4. Vergnaud AC, Bertrais S, Oppert JM, Maillard-Teyssier L, Galan P, Hercberg $S$, et al. Weight fluctuations and risk for metabolic syndrome in an adult cohort. Int J Obes. 2008;32(2):315-21.

5. Lahmann PH, Lissner L, Gullberg B, Berglund G. Sociodemographic factors associated with long-term weight gain, current body fatness and central adiposity in Swedish women. International. Int J Obes Relat Metab Disord. 2000; 24(6):685-94.

6. Chor D, Faerstein E, Kaplan GA, Lynch JW, Lopes CS. Association of weight change with ethnicity and life course socioeconomic position among Brazilian civil servants. Int J Epidemiol. 2004;33(1):100-6.

7. Monteiro CA, Moura EC, Conde WL, Popkin BM. Socioeconomic status and obesity in adult populations of developing countries: a review. Bull World Health Org. 2004;82(12):940-6.

8. Sichieri R, Silva CV, Moura AS. Combined effect of short stature and socioeconomic status on body mass index and weight gain during reproductive age in Brazilian women. Braz J Med Biol Res. 2003;36(10):1319-25. 
9. Monteiro CA, de Moura EC, Jaime PC, Lucca A, Florindo AA, Figueiredo IC. Surveillance of risk factors for chronic diseases through telephone interviews. Rev Saúde Pública. 2005;39(1):47-57.

10. MartinsTG, de Assis MAA, Nahas MV, Gauche H, Moura EC. Inatividade física no lazer de adultos e fatores associados. Rev Saúde Pública. 2009; 43(5):814-24.

11. Monteiro CA, Florindo AA, Claro RM, Moura EC. Validade de indicadores de atividade física e sedentarismo obtidos por inquérito telefônico. Rev Saúde Pública. 2008;42(4):575-81.

12. Monteiro CA, Moura EC, Jaime PC, Claro RM. Validade de indicadores do consumo de alimentos e bebidas obtidos por inquérito telefônico. Rev Saúde Pública. 2008;42(4):582-9.

13. Brasil. Ministério da Saúde. Guia Alimentar da População Brasileira. Brasília, DF; 2004.

14. Brasil. Ministério da Saúde. Análise da estratégia global para alimentação saudável, Atividade Física e Saúde. Brasília, DF. 2004.

15. Sandsquist J, Johansson S-E. The influence of socioeconomic status, ethnicity and lifestyle on body mass index in a longitudinal study. Int J Epidemiol. 1998;27(1):57-63.

16. Ball K, Brown W, Crawford D. Who does not gain weight? Prevalence and predictors of weight maintenance in young women. Int J Obes Relat Metab Disord. 2002;26(12):1570-8.

17. Victora CG, Huttly SR, Fuchs SC, Olinto MT.The role of conceptual frameworks in epidemiological analysis: a hierarchical approach. Int J Epidemiol. 1997;26(1):224-7.

18. Yarnell JWG, Patterson CC, Thomas HF, Sweetnam PM. Comparison of weight in middle age, weight at 18 years, and weight change between, in predicting subsequent 14 year mortality and coronary events: Caerphilly Prospective Study. J Epidemiol Community Health. 2000;54(5):344-8.

19. van Lenthe FJ, Droomers M, Schrijvers CTM, Mackenbach JP. Socio-demographic variables and 6 year change in body mass index: longitudinal results from the GLOBE study. Int $\mathrm{J}$ Obes. 2000;24:1077-84.

20. Truong KD, Sturm R. Weight gain trends across sociodemographic groups in the United States. Am J Public Health. 2005;95(9):1602-06.

21. Peixoto M do R, Benício MH, Jardim PC. Validade do peso e da altura auto-referidos: o estudo de Goiânia. Rev Saúde Pública. 2006;40(6):1065-72.

22. Moura EC, Morais Neto OL, Malta DC, Moura L, Silva NN, Bernal $\mathrm{R}$, et al. Vigilância de Fatores de Risco para Doenças Crônicas por Inquérito Telefônico nas capitais dos 26 estados brasileiros e no Distrito Federal (2006). Rev Bras Epidemiol. 2008;11(supl 1):20-37.

23. Bell AC, Ge K, Popkin BM. Weight gain and its predictors in Chinese adults. Int J Obes Relat Metab Disord. 2001;25(7):1079-86.

24. Vioque J, WeinbrennerT, Castelló A, Asensio L, Garcia de la Hera $M$. Intake of fruits and vegetables in relation to 10 -year weight gain among Spanish adults. Obesity. 2008;16(3):664-70.

25. Newby PK, Muller D, Hallfrisch J, Qiao N, Andres R, Tucker KL. Dietary patterns and changes in body mass index and waist circumference in adults. Am J Clin Nutr. 2003;77(6):1417-25.

26. Togo $\mathrm{P}$, Osler M, Sørensen TI, Heitmann BL. Food intake patterns and body mass index in observational studies. Int $\mathrm{J}$ Obes Relat Metab Disord. 2001;25(12):1741-51.

27. Perozzo G, Olinto MTA, Dias-da-Costa JS, Henn RL, Sarriera J, Pattussi MP. Associação dos padrões alimentares com obesidade geral e abdominal em mulheres residentes no Sul do Brasil. Cad Saúde Pública. 2008;24(10):2427-39.

28. Brasil. Ministério da Saúde. Vigitel Brasil 2007: vigilância de fatores de risco e proteção para doenças crônicas por inquérito telefônico / Ministério da Saúde, Secretaria de Vigilância em Saúde, Secretaria de Gestão Estratégica e Participativa. Brasília: Ministério da Saúde; 2008. 